\title{
Chemoselective Suzuki Cross-Coupling Reactions of Chiral Pyrrolizines
}

\author{
Hyo-Jun Lee and Chang-Woo Cho* \\ Department of Chemistry, Kyungpook National University, Daegu 702-701, Korea. *E-mail: cwcho@knu.ac.kr \\ Received November 13, 2010, Accepted November 18, 2010
}

Key Words: Chemoselective, Suzuki cross-coupling, Pyrrolizine, Sequential, Unsymmetrical

Pyrrolizines have potent cytostatic effects and are thus potentially useful for the development of antitumor and antiviral agents. ${ }^{1}$ In particular, chiral trifluoromethylated pyrrolizines would play a unique and significant role in medicinal chemistry because the introduction of a trifluoromethyl group into pharmaceuticals often enhances their physical and/or chemical properties. ${ }^{2}$ Recently, we reported that the enantio- and diastereoselective organocatalytic cascade conjugate addition-aldol reactions of a series of 2-trifluoroacetylpyrroles to $\alpha, \beta$-unsaturated aldehydes afford highly functionalized chiral pyrrolizines that bear a trifluoromethyl group at the stereogenic center in good yields, high enantioselectivities, and excellent diastereoselectivities. ${ }^{3}$ Using the chiral pyrrolizines as the cascade products, we planned the development of an efficient synthesis of unsymmetrically diarylsubstituted chiral pyrrolizines via two sequential Suzuki cross-coupling reactions with different arylboronic acids in a highly chemoselective controlled manner. ${ }^{4}$ Specifically, in these two sequential Suzuki couplings of multiple halogenated heterocycles, ${ }^{5}$ the use of pyrrolizines remains unexplored, in spite of the importance of pyrrolizines as pharmacophores in biologically active compounds. Here, we report the development of the efficient synthetic route of a series of unsymmetrically 5,6-diarylsubstituted chiral pyrrolizines via chemoselective Suzuki cross-coupling reactions of chiral 6-bromo5-iodopyrrolizines, followed by the sequential 2nd Suzuki cross-coupling reaction (Scheme 1).

To explore the feasibility of chemoselective Suzuki crosscoupling reactions of 6-bromo-5-iodopyrrolizines, prepared according to our previous paper, ${ }^{3}$ with various arylboronic acids, the Suzuki couplings of 6-bromo-5-iodopyrrolizine 1a with 3-nitrophenylboronic acid (2) were carried out in the presence of $\mathrm{Pd}\left(\mathrm{PPh}_{3}\right)_{4}$ as the catalyst and $\mathrm{K}_{2} \mathrm{CO}_{3}$ as the base in 1,4dioxane- $\mathrm{H}_{2} \mathrm{O}$ solution at elevated temperature (Table 1). The initial cross-couplings attempts using $\mathrm{Pd}\left(\mathrm{PPh}_{3}\right)_{4}(5 \mathrm{~mol} \%)$ and $\mathrm{K}_{2} \mathrm{CO}_{3}(200 \mathrm{~mol} \%)$ in 1,4-dioxane $(0.2 \mathrm{M})-\mathrm{H}_{2} \mathrm{O}(1.0 \mathrm{M})$ solution at $60{ }^{\circ} \mathrm{C}$ chemoselectively afforded the desired coupling product, 6-bromo-5-(3-nitrophenyl)pyrrolizines 3, in 44\% yield,

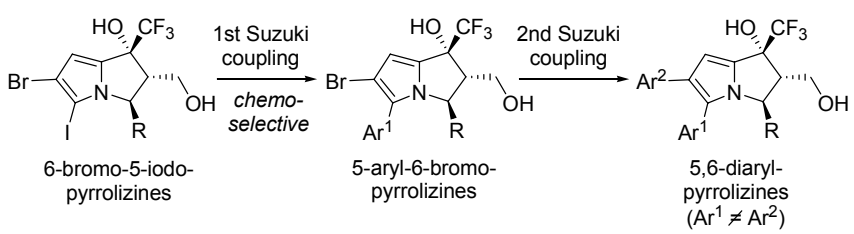

Scheme 1. Synthetic approach to unsymmetrically 5,6-diarylsubstituted chiral pyrrolizines via two sequential Suzuki cross-coupling reactions without 5-iodo-6-(3-nitrophenyl)pyrrolizine as the by-product (Table 1, entry 1). The chemoselective coupling product 3 was identified by ${ }^{1} \mathrm{H}$ NMR and high-resolution mass spectroscopy analyses. At $80^{\circ} \mathrm{C}$, the coupling product 3 showed an increased yield of $57 \%$ (Table 1, entry 2). Furthermore, when the loading of $\mathrm{Pd}\left(\mathrm{PPh}_{3}\right)_{4}$ was increased to $10 \mathrm{~mol} \%$, the coupling product 3 was obtained in $63 \%$ yield (Table 1 , entry 3 ). Remarkably, when the concentration of the 1,4-dioxane- $\mathrm{H}_{2} \mathrm{O}$ co-solvent was decreased to $0.1 \mathrm{M}$ and $0.15 \mathrm{M}$, respectively, with the other reaction conditions remaining the same, the cross-coupling reaction afforded the coupling product 3 in 74\% yield (Table 1, entry 4). At higher temperatures, under otherwise identical conditions, the coupling product $\mathbf{3}$ was obtained in a decreased yield of 56\% (Table 1, entry 5).

In addition, we applied the optimized conditions of the chemoselective Suzuki cross-coupling reaction of 6-bromo-5-iodopyrrolizine 1a to the site-selective Suzuki cross-coupling reaction $^{6}$ of 5,6-dibromopyrrolizine 4 , generally controlled by electronic and steric parameters, to obtain the desired product, 6-bromo-5-(3-nitrophenyl)pyrrolizines 3; this product is the same as that obtained from chemoselective couplings (Scheme 2). However, the site-selective cross-coupling reaction afforded the desired coupling product 3 and the symmetrical 5,6-diarylpyrrolizine $\mathbf{5}$ as the by-product in low selectivity and poor yields. Hence, the chemoselective couplings proved superior in the case of 6-bromo-5-iodopyrrolizine 1 a.

Table 1. Optimization of chemoselective Suzuki cross-coupling reactions of 6-bromo-5-iodopyrrolizine 1a with 3-nitrophenylboronic $\operatorname{acid}(\mathbf{2})^{a}$

entry
$\mathbf{1 a}(100 \mathrm{~mol} \%)$

$\overline{{ }^{a} \text { Procedure: To a 1,4-dioxane }(0.1 \mathrm{M}) \text { of } \mathbf{1 a}(100 \mathrm{~mol} \%) \text { was added } \mathrm{Pd}\left(\mathrm{PPh}_{3}\right)_{4}}$ $(10 \mathrm{~mol} \%)$ at rt. After stirring for $20 \mathrm{~min}, \mathrm{~K}_{2} \mathrm{CO}_{3}(200 \mathrm{~mol} \%)$ in $\mathrm{H}_{2} \mathrm{O}$ $(0.15 \mathrm{M})$ and arylboronic acid $(110 \mathrm{~mol} \%)$ were added. The mixture was stirred at 60,80 , or $100^{\circ} \mathrm{C}$ for $12 \mathrm{~h}$. 


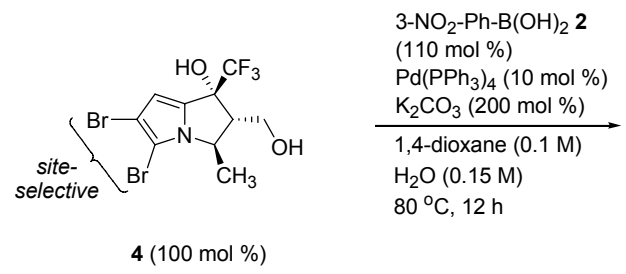

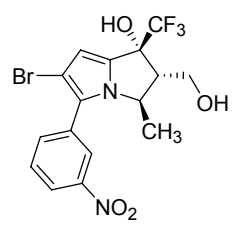

3, $16 \%$ yield

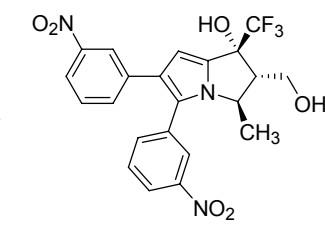

5, $14 \%$ yield

Scheme 2. Site-selective Suzuki cross-coupling reaction of 5,6-dibromopyrrolizine 4 with 3-nitrophenylboronic acid (2)

Table 2. Chemoselective Suzuki cross-coupling reactions of 6-bromo5-iodopyrrolizines 1 with various boronic acids ${ }^{a}$

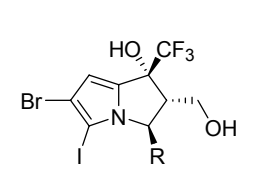

$$
\mathrm{Ar}^{1} \mathrm{~B}(\mathrm{OH})_{2}(110 \mathrm{~mol} \%)
$$
$\mathrm{Pd}\left(\mathrm{PPh}_{3}\right)_{4}(10 \mathrm{~mol} \%)$ $\mathrm{K}_{2} \mathrm{CO}_{3}(200 \mathrm{~mol} \%)$

1,4-dioxane $(0.1 \mathrm{M})$ $\mathrm{H}_{2} \mathrm{O}(0.15 \mathrm{M})$

1a, $\mathrm{R}=\mathrm{CH}_{3}$ $80^{\circ} \mathrm{C}, 12 \mathrm{~h}$

1c, $\mathrm{R}=\mathrm{CH}_{2} \mathrm{OCH}_{3}$

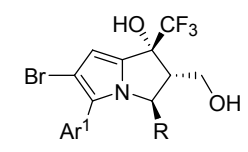

3, 6-10

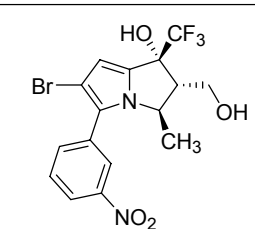

3, $74 \%$ yield<smiles>CCCC1[C@H](CO)[C@@](O)(C(F)(F)F)c2cc(Br)c(-c3ccc(C)cc3)n21</smiles>

8, $70 \%$ yield

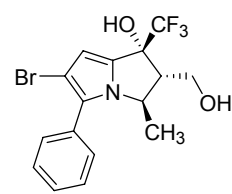

6, $81 \%$ yield

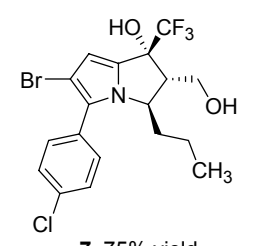

7, $75 \%$ yield
${ }^{a}$ Procedure: See the Experimental Section. ${ }^{b} 1.5$ eq of 2,4-difluorophenylboronic acid was used.

To expand the scope of the chemoselective Suzuki crosscoupling reactions of 6-bromo-5-iodopyrrolizines 1a-1c, which bear aliphatic and methyl-protected hydroxymethyl substituents, the cross-couplings of the same compounds with a series of arylboronic acids having various electron-withdrawing and electron-donating substituents were explored under the optimized reaction conditions (Table 2). The corresponding coupling products 3 and 6-10 were chemoselectively obtained in high yields.

Next, we further explored the sequential 2nd Suzuki crosscoupling reactions of chiral 5-aryl-6-bromopyrrolizines with various arylboronic acids to obtain unsymmetrically 5,6-diarylsubstituted chiral pyrrolizines (Scheme 3). As a representative substrate in the 2nd Suzuki cross-coupling reactions, 6-bromo5-(3-nitrophenyl)pyrrolizines 3 underwent the cross-couplings with a series of arylboronic acids ( $150 \mathrm{~mol} \%$ ) bearing electron-withdrawing and electron-donating substituents in the presence of $\mathrm{Pd}\left(\mathrm{PPh}_{3}\right)_{4}(10 \mathrm{~mol} \%)$ and $\mathrm{K}_{2} \mathrm{CO}_{3}(200 \mathrm{~mol} \%)$ in 1,4-dioxane $(0.1 \mathrm{M})-\mathrm{H}_{2} \mathrm{O}(0.15 \mathrm{M})$ solution at $100{ }^{\circ} \mathrm{C}$ to provide the desired unsymmetrical 5,6-diarylpyrrolizines 11-14 in high yields.

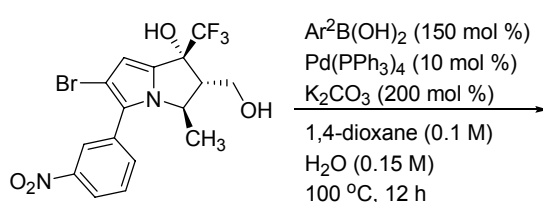

3

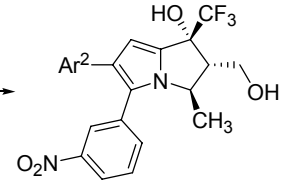

$11, \mathrm{Ar}^{2}=\mathrm{Ph}, 83 \%$ yield $12, \mathrm{Ar}^{2}=4-\mathrm{Cl}-\mathrm{Ph}, 87 \%$ yield $13, \mathrm{Ar}^{2}=4-\mathrm{CH}_{3}-\mathrm{Ph}, 84 \%$ yield $14, \mathrm{Ar}^{2}=4-\mathrm{CH}_{3} \mathrm{O}-\mathrm{Ph}, 86 \%$ yield

Scheme 3. Synthesis of unsymmetrical diarylpyrrolizines 11-14 via the sequential 2nd Suzuki couplings of 6-bromo-5-(3-nitrophenyl) pyrrolizines $\mathbf{3}$ with various arylboronic acids

In summary, the chemoselective Suzuki cross-coupling reaction between chiral 6-bromo-5-iodopyrrolizines and a series of arylboronic acids has been achieved using $\mathrm{Pd}\left(\mathrm{PPh}_{3}\right)_{4}$ as the catalyst and $\mathrm{K}_{2} \mathrm{CO}_{3}$ as the base in 1,4-dioxane- $\mathrm{H}_{2} \mathrm{O}$ solution. The cross-coupling reaction provides chemoselectively various chiral 5-aryl-6-bromopyrrolizines in high yields. The sequential 2nd Suzuki cross-coupling reaction of 6-bromo-5-(3-nitrophenyl)pyrrolizines with arylboronic acids bearing electronwithdrawing and electron-donating substituents affords a variety of unsymmetrically 5,6-diarylsubstituted chiral pyrrolizines in high yields. In addition, this is the first example of two sequential Suzuki cross-coupling reactions of dihalopyrrolizines to provide unsymmetrical diarylpyrrolizines. Further studies on the development of two sequential Suzuki cross-coupling reactions are underway.

\section{Experimental Section}

General Procedure for the Chemoselective Suzuki Crosscoupling Reactions. To a 1,4-dioxane solution $(0.1 \mathrm{M})$ of 6bromo-5-iodopyrrolizine $1 \mathbf{a}(0.2 \mathrm{mmol}, 100 \mathrm{~mol} \%)$ was added $\mathrm{Pd}\left(\mathrm{PPh}_{3}\right)_{4}(0.02 \mathrm{mmol}, 10 \mathrm{~mol} \%)$ at $\mathrm{rt}$ under argon atmosphere. After stirring for $20 \mathrm{~min}, \mathrm{~K}_{2} \mathrm{CO}_{3}(0.4 \mathrm{mmol}, 200 \mathrm{~mol} \%)$ in $\mathrm{H}_{2} \mathrm{O}$ $(0.15 \mathrm{M})$ and arylboronic acid $(0.22 \mathrm{mmol}, 110 \mathrm{~mol} \%)$ were added. The mixture was stirred at $80^{\circ} \mathrm{C}$ for $12 \mathrm{~h}$. After cooling to rt, the mixture was diluted with EtOAc and washed with saturated $\mathrm{NaHCO}_{3}$ followed by brine. And then, the mixture was dried over $\mathrm{MgSO}_{4}$ and filtered through a short Celite pad. The solution was concentrated in vacuo and the residue was purified by flash column chromatography to provide the desired product, 5-aryl-6-bromopyrrolizine.

General Procedure for the Sequential 2nd Suzuki Cross-coupling Reactions. To a 1,4-dioxane solution ( $0.1 \mathrm{M})$ of 6-bromo5-(3-nitrophenyl)pyrrolizine $3(0.2 \mathrm{mmol}, 100 \mathrm{~mol} \%)$ was added $\mathrm{Pd}\left(\mathrm{PPh}_{3}\right)_{4}(0.02 \mathrm{mmol}, 10 \mathrm{~mol} \%)$ at rt under argon atmosphere. 
After stirring for $20 \mathrm{~min}, \mathrm{~K}_{2} \mathrm{CO}_{3}(0.4 \mathrm{mmol}, 200 \mathrm{~mol} \%)$ in $\mathrm{H}_{2} \mathrm{O}$ $(0.15 \mathrm{M})$ and arylboronic acid $(0.3 \mathrm{mmol}, 150 \mathrm{~mol} \%)$ were added. The mixture was stirred at $100{ }^{\circ} \mathrm{C}$ for $12 \mathrm{~h}$. After cooling to rt, the mixture was diluted with EtOAc and washed with saturated $\mathrm{NaHCO}_{3}$ followed by brine. And then, the mixture was dried over $\mathrm{MgSO}_{4}$ and filtered through a short Celite pad. The solution was concentrated in vacuo and the residue was purified by flash column chromatography to provide the desired product, 6-aryl-5-(3-nitrophenyl)pyrrolizine.

The Spectroscopic Data of 3 and 5-14 are as Follows.

Compound 3: yellow solid, mp $123-124^{\circ} \mathrm{C}$; IR (neat) 3446, 2948, 1716, 1533, 1349, 1283, 1179, 1081, $740 \mathrm{~cm}^{-1} ;{ }^{1} \mathrm{H}$ NMR $\left(400 \mathrm{MHz}, \mathrm{CDCl}_{3}\right) \delta 8.35-8.34(\mathrm{~m}, 1 \mathrm{H}), 8.25-8.22(\mathrm{~m}, 1 \mathrm{H})$, 7.84-7.82 (m, 1H), $7.64(\mathrm{t}, J=8.0 \mathrm{~Hz}, 1 \mathrm{H}), 6.36(\mathrm{~s}, 1 \mathrm{H}), 4.89$ $4.83(\mathrm{~m}, 1 \mathrm{H}), 4.23-4.20(\mathrm{~m}, 1 \mathrm{H}), 4.00-3.94(\mathrm{~m}, 1 \mathrm{H}), 3.63(\mathrm{~s}$, $1 \mathrm{H}), 2.75-2.71(\mathrm{~m}, 1 \mathrm{H}), 2.48-2.45(\mathrm{~m}, 1 \mathrm{H}), 1.11(\mathrm{~d}, J=6.4 \mathrm{~Hz}$, $3 \mathrm{H}) ;{ }^{13} \mathrm{C}$ NMR $\left(100 \mathrm{MHz}, \mathrm{CDCl}_{3}\right) \delta 148.2,135.1,133.4,131.9$, $129.6,125.1,123.8,122.7,124.4\left(\mathrm{q},{ }^{1} J_{C F}=278.0 \mathrm{~Hz}\right), 105.7$, $100.4,77.6\left(\mathrm{q},{ }^{2} J_{C F}=32.0 \mathrm{~Hz}\right), 59.8,55.8,55.1,20.4$; HRMS calcd for [M] $\mathrm{C}_{16} \mathrm{H}_{14} \mathrm{BrF}_{3} \mathrm{~N}_{2} \mathrm{O}_{4} 434.0089$, found 434.0091 .

Compound 5: light yellow solid, mp $82-83{ }^{\circ} \mathrm{C}$; IR (neat) $3423,2930,1734,1708,1532,1349,1160,1082,741 \mathrm{~cm}^{-1} ;{ }^{1} \mathrm{H}$ NMR (400 MHz, $\left.\mathrm{CDCl}_{3}\right) \delta$ 8.25-8.22 (m, 1H), 8.19-8.18 (m, $1 \mathrm{H}), 8.01-7.98(\mathrm{~m}, 2 \mathrm{H}), 7.62-7.55(\mathrm{~m}, 2 \mathrm{H}), 7.40-7.32(\mathrm{~m}, 2 \mathrm{H})$, $6.46(\mathrm{~s}, 1 \mathrm{H}), 4.89-4.83(\mathrm{~m}, 1 \mathrm{H}), 4.26-4.22(\mathrm{~m}, 1 \mathrm{H}), 4.03-3.97$ $(\mathrm{m}, 1 \mathrm{H}), 3.92(\mathrm{~s}, 1 \mathrm{H}), 2.82-2.79(\mathrm{~m}, 1 \mathrm{H}), 2.72-2.69(\mathrm{~m}, 1 \mathrm{H})$, $1.10(\mathrm{~d}, J=6.4 \mathrm{~Hz}, 3 \mathrm{H}) ;{ }^{13} \mathrm{C} \mathrm{NMR}\left(100 \mathrm{MHz}, \mathrm{CDCl}_{3}\right) \delta 148.4$, 148.2, 137.0, 135.8, 134.2, 133.9, 133.0, 130.0, 129.3, 126.6, $124.6\left(\mathrm{q},{ }^{1} J_{C F}=281.0 \mathrm{~Hz}\right), 124.5,124.1,123.0,122.6,120.9$, 102.7, $77.2\left(\mathrm{q},{ }^{2} J_{C F}=32.0 \mathrm{~Hz}\right), 59.9,55.6,54.8,20.6$; HRMS calcd for [M] $\mathrm{C}_{22} \mathrm{H}_{18} \mathrm{~F}_{3} \mathrm{~N}_{3} \mathrm{O}_{6} 477.1148$, found 477.1151 .

Compound 6: colorless oil; IR (neat) 3399, 2933, 1708, $1468,1284,1175,1079,701 \mathrm{~cm}^{-1} ;{ }^{1} \mathrm{H}$ NMR $\left(400 \mathrm{MHz}, \mathrm{CDCl}_{3}\right)$

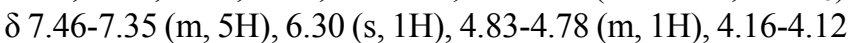
$(\mathrm{m}, 1 \mathrm{H}), 3.94(\mathrm{~s}, 1 \mathrm{H}), 3.93-3.87(\mathrm{~m}, 1 \mathrm{H}), 2.85-2.82(\mathrm{~m}, 1 \mathrm{H})$, $2.69-2.66(\mathrm{~m}, 1 \mathrm{H}), 1.06(\mathrm{~d}, J=6.0 \mathrm{~Hz}, 3 \mathrm{H}) ;{ }^{13} \mathrm{C} \mathrm{NMR}(100 \mathrm{MHz}$, $\left.\mathrm{CDCl}_{3}\right) \delta 131.9,130.2,129.2,128.4,128.0,127.8,124.5(\mathrm{q}$, $\left.{ }^{1} J_{C F}=284.0 \mathrm{~Hz}\right), 105.1,98.9,77.6\left(\mathrm{q},{ }^{2} J_{C F}=32.0 \mathrm{~Hz}\right), 59.8,55.5$, 55.2, 20.1; HRMS calcd for [M] $\mathrm{C}_{16} \mathrm{H}_{15} \mathrm{BrF}_{3} \mathrm{NO}_{2} 389.0238$, found 389.0239 .

Compound 7: colorless oil; IR (neat) 3399, 2961, 1709, 1466, 1276, 1182, 1092, 833, $722 \mathrm{~cm}^{-1}$; ${ }^{1} \mathrm{H} \mathrm{NMR}(400 \mathrm{MHz}$, $\left.\mathrm{CDCl}_{3}\right) \delta$ 7.42-7.05 (m, 5H), $6.30(\mathrm{~s}, 1 \mathrm{H}), 4.70-4.66(\mathrm{~m}, 1 \mathrm{H})$, 4.14-4.09 (m, 1H), 3.89-3.83 (m, 1H), $3.85(\mathrm{~s}, 1 \mathrm{H}), 2.89-2.83$ $(\mathrm{m}, 2 \mathrm{H}), 1.43-1.30(\mathrm{~m}, 2 \mathrm{H}), 1.19-1.06(\mathrm{~m}, 1 \mathrm{H}), 1.04-0.90(\mathrm{~m}$, $1 \mathrm{H}), 0.67(\mathrm{t}, J=7.6 \mathrm{~Hz}, 3 \mathrm{H}) ;{ }^{13} \mathrm{C} \mathrm{NMR}\left(100 \mathrm{MHz}, \mathrm{CDCl}_{3}\right) \delta$ $134.0,132.6,130.2,128.8,128.7,126.5,124.5\left(\mathrm{q},{ }^{1} J_{C F}=282.0\right.$ $\mathrm{Hz}), 105.3,99.4,77.7\left(\mathrm{q},{ }^{2} J_{C F}=32.0 \mathrm{~Hz}\right), 60.9,59.2,51.8,34.9$, 16.7, 13.5; HRMS calcd for [M] $\mathrm{C}_{18} \mathrm{H}_{18} \mathrm{BrClF}_{3} \mathrm{NO}_{2} 451.0162$, found 451.0160 .

Compound 8: colorless oil; IR (neat) 3419, 2961, 1709, 1437, $1279,1176,1083,822,722 \mathrm{~cm}^{-1} ;{ }^{1} \mathrm{H}$ NMR $\left(400 \mathrm{MHz}, \mathrm{CDCl}_{3}\right)$

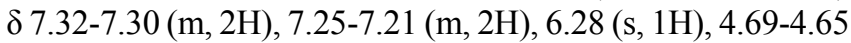
$(\mathrm{m}, 1 \mathrm{H}), 4.13-4.06(\mathrm{~m}, 1 \mathrm{H}), 4.01(\mathrm{~s}, 1 \mathrm{H}), 3.88-3.81(\mathrm{~m}, 1 \mathrm{H})$, $2.90-2.85(\mathrm{~m}, 2 \mathrm{H}), 2.39$ (s, 3H), 1.38-1.21 (m, 2H), 1.14-1.04 $(\mathrm{m}, 1 \mathrm{H}), 1.03-0.92(\mathrm{~m}, 1 \mathrm{H}), 0.64(\mathrm{t}, J=7.2 \mathrm{~Hz}, 3 \mathrm{H}) ;{ }^{13} \mathrm{C} \mathrm{NMR}$ $\left(100 \mathrm{MHz} \mathrm{CDCl}_{3}\right) \delta 137.9,131.9,129.2,128.8,127.9,127.2$, $126.0,124.6\left(\mathrm{q},{ }^{1} J_{C F}=281.0 \mathrm{~Hz}\right), 123.2,104.9,98.7,77.4(\mathrm{q}$, $\left.{ }^{2} J_{C F}=32.0 \mathrm{~Hz}\right), 60.9,59.1,51.8,34.6,21.3,16.6,13.5 ;$ HRMS calcd for [M] $\mathrm{C}_{19} \mathrm{H}_{21} \mathrm{BrF}_{3} \mathrm{NO}_{2}$ 431.0708, found 431.0706.

Compound 9: colorless oil; IR (neat) 3422, 2937, 1709, 1614, $1476,1287,1250,1176,1033,836,726 \mathrm{~cm}^{-1}$; ${ }^{1} \mathrm{H}$ NMR (400 $\left.\mathrm{MHz}, \mathrm{CDCl}_{3}\right) \delta$ 7.39-7.35 (m, 2H), 6.99-6.95 (m, 2H), $6.30(\mathrm{~s}$, $1 \mathrm{H}), 4.78-4.74(\mathrm{~m}, 1 \mathrm{H}), 4.15-4.12(\mathrm{~m}, 1 \mathrm{H}), 3.93-3.87(\mathrm{~m}, 1 \mathrm{H})$, $3.86(\mathrm{~s}, 3 \mathrm{H}), 3.69(\mathrm{~s}, 1 \mathrm{H}), 3.24(\mathrm{dd}, J=10.0,3.2 \mathrm{~Hz}, 1 \mathrm{H}), 3.19$ $3.16(\mathrm{~m}, 1 \mathrm{H}), 3.08(\mathrm{~s}, 3 \mathrm{H}), 3.04(\mathrm{dd}, J=9.6,6.8 \mathrm{~Hz}, 1 \mathrm{H}), 2.79$ $(\mathrm{dd}, J=8.0,3.6 \mathrm{~Hz}, 1 \mathrm{H}) ;{ }^{13} \mathrm{C} \mathrm{NMR}\left(100 \mathrm{MHz}, \mathrm{CDCl}_{3}\right) \delta 159.9$, $132.8,130.9,128.2,125.1\left(\mathrm{q},{ }^{1} J_{C F}=280.0 \mathrm{~Hz}\right), 122.8,114.4$, $105.7,99.3,77.7\left(\mathrm{q},{ }^{2} J_{C F}=32.0 \mathrm{~Hz}\right), 71.3,60.9,59.4,59.3$, 55.7, 50.9; HRMS calcd for [M] $\mathrm{C}_{18} \mathrm{H}_{19} \mathrm{BrF}_{3} \mathrm{NO}_{4} 449.0450$, found 449.0420 .

Compound 10: light yellow oil; IR (neat) 3410, 2932, 1709, $1595,1464,1282,1176,954,852,729 \mathrm{~cm}^{-1} ;{ }^{1} \mathrm{H}$ NMR (400 $\left.\mathrm{MHz}, \mathrm{CDCl}_{3}\right) \delta$ 7.46-7.40 (m, 1H), 7.02-6.91 (m, 2H), $6.34(\mathrm{~s}$, $1 \mathrm{H}), 4.70-4.66(\mathrm{~m}, 1 \mathrm{H}), 4.21(\mathrm{~s}, 1 \mathrm{H}), 4.12(\mathrm{dd}, J=11.6,3.2 \mathrm{~Hz}$, $1 \mathrm{H}), 3.91-3.87(\mathrm{~m}, 2 \mathrm{H}), 3.27(\mathrm{dd}, J=10.0,3.6 \mathrm{~Hz}, 1 \mathrm{H}), 3.15-$ $3.10(\mathrm{~m}, 2 \mathrm{H}), 3.06(\mathrm{~s}, 3 \mathrm{H}), 2.84-2.76(\mathrm{~m}, 1 \mathrm{H}) ;{ }^{13} \mathrm{C} \mathrm{NMR}(100$ $\left.\mathrm{MHz} \mathrm{CDCl}_{3}\right) \delta 163.2\left(\mathrm{dd},{ }^{1} J_{C F}=250.0 \mathrm{~Hz},{ }^{3} J_{C F}=12.0 \mathrm{~Hz}\right)$, $160.3\left(\mathrm{dd},{ }^{1} J_{C F}=249.0 \mathrm{~Hz},{ }^{3} J_{C F}=12.0 \mathrm{~Hz}\right), 133.4,133.3,124.4$ $\left(\mathrm{q},{ }^{1} J_{C F}=280.0 \mathrm{~Hz}\right), 121.2,114.4\left(\mathrm{dd},{ }^{2} J_{C F}=16.0 \mathrm{~Hz},{ }^{4} J_{C F}=4.0\right.$ $\mathrm{Hz}), 111.6\left(\mathrm{dd},{ }^{2} J_{C F}=22.0 \mathrm{~Hz},{ }^{4} J_{C F}=4.0 \mathrm{~Hz}\right), 105.4,104.3(\mathrm{dd}$, $\left.{ }^{2} J_{C F}=26.0 \mathrm{~Hz},{ }^{2} J_{C F}=25.0 \mathrm{~Hz}\right), 101.0,77.6\left(\mathrm{q},{ }^{2} J_{C F}=32.0 \mathrm{~Hz}\right)$, 71.0, 60.2, 59.7, 58.9, 50.1; HRMS calcd for $[\mathrm{M}] \mathrm{C}_{17} \mathrm{H}_{15} \mathrm{BrF}_{5} \mathrm{NO}_{3}$ 455.0155 , found 455.0156 .

Compound 11: yellow solid, mp $171-172{ }^{\circ} \mathrm{C}$; IR (neat) 3419, 2933, 1735, 1532, 1351, 1287, 1151, 1107, 950, 772, $702 \mathrm{~cm}^{-1}$; ${ }^{1} \mathrm{H}$ NMR $\left(400 \mathrm{MHz}, \mathrm{CDCl}_{3}\right) \delta 8.19-8.16(\mathrm{~m}, 2 \mathrm{H})$, 7.60-7.58 (m, 1H), 7.51-7.47 (m, 1H), 7.24-7.17 (m, 3H), 7.14$7.12(\mathrm{~m}, 2 \mathrm{H}), 4.90-4.83(\mathrm{~m}, 1 \mathrm{H}), 4.25-4.20(\mathrm{~m}, 1 \mathrm{H}), 4.03-3.96$ $(\mathrm{m}, 1 \mathrm{H}), 3.41(\mathrm{~s}, 1 \mathrm{H}), 2.81-2.77(\mathrm{~m}, 1 \mathrm{H}), 2.54(\mathrm{dd}, J=7.6,4.4 \mathrm{~Hz}$, 1H), $1.09(\mathrm{~d}, J=6.4 \mathrm{~Hz}, 3 \mathrm{H}) ;{ }^{13} \mathrm{C} \mathrm{NMR}\left(100 \mathrm{MHz}, \mathrm{CDCl}_{3}\right) \delta$ $148.3,135.9,135.2,133.8,133.6,129.6,129.3,128.4,128.2$, $126.3,124.6\left(\mathrm{q},{ }^{1} J_{C F}=279.0 \mathrm{~Hz}\right), 124.0,123.8,122.3,102.9$, $77.3\left(\mathrm{q},{ }^{2} J_{C F}=32.0 \mathrm{~Hz}\right), 60.0,55.8,54.7,20.7$; HRMS calcd for $[\mathrm{M}] \mathrm{C}_{22} \mathrm{H}_{19} \mathrm{~F}_{3} \mathrm{~N}_{2} \mathrm{O}_{4} 432.1297$, found 432.1298 .

Compound 12: yellow solid, mp $148-149^{\circ} \mathrm{C}$; IR (neat) 3418, 2888, 1734, 1533, 1497, 1349, 1168, 1152, 1045, 950, 838, 725 $\mathrm{cm}^{-1}$; ${ }^{1} \mathrm{H}$ NMR $\left(400 \mathrm{MHz}, \mathrm{CDCl}_{3}\right) \delta 8.19-8.16(\mathrm{~m}, 2 \mathrm{H}), 7.58-$ $7.49(\mathrm{~m}, 2 \mathrm{H}), 7.17-7.15(\mathrm{~m}, 2 \mathrm{H}), 7.04-7.02(\mathrm{~m}, 2 \mathrm{H}), 6.34(\mathrm{~s}$, $1 \mathrm{H}), 4.87-4.80(\mathrm{~m}, 1 \mathrm{H}), 4.22-4.19(\mathrm{~m}, 1 \mathrm{H}), 4.01-3.95(\mathrm{~m}, 1 \mathrm{H})$, $3.84(\mathrm{~s}, 1 \mathrm{H}), 2.79-2.77(\mathrm{~m}, 2 \mathrm{H}), 1.08(\mathrm{~d}, J=6.4 \mathrm{~Hz}, 3 \mathrm{H}) ;{ }^{13} \mathrm{C}$ NMR $\left(100 \mathrm{MHz}, \mathrm{CDCl}_{3}\right) \delta 148.4,135.9,133.8,133.7,133.5$, $132.1,129.7,129.4,128.6,128.0,124.6\left(\mathrm{q},{ }^{1} J_{C F}=281.0 \mathrm{~Hz}\right)$, $124.0,123.9,122.5,102.7,77.3\left(\mathrm{q},{ }^{2} J_{C F}=31.0 \mathrm{~Hz}\right), 59.9,55.7$, 54.7, 20.6; HRMS calcd for [M] $\mathrm{C}_{22} \mathrm{H}_{18} \mathrm{ClF}_{3} \mathrm{~N}_{2} \mathrm{O}_{4} 466.0907$, found 466.0904 .

Compound 13: yellow solid, mp 90 - $91^{\circ} \mathrm{C}$; IR (neat) 3394, 2888, 1532, 1349, 1285, 1170, 1152, 1043, 950, 797, $695 \mathrm{~cm}^{-1}$; ${ }^{1} \mathrm{H}$ NMR (400 MHz, $\left.\mathrm{CDCl}_{3}\right) \delta 8.19-8.14(\mathrm{~m}, 2 \mathrm{H}), 7.60-7.57$ $(\mathrm{m}, 1 \mathrm{H}), 7.51-7.47(\mathrm{~m}, 1 \mathrm{H}), 7.03-6.99(\mathrm{~m}, 4 \mathrm{H}), 6.35(\mathrm{~s}, 1 \mathrm{H})$, 4.88-4.82 (m, 1H), 4.23-4.18 (m, 1H), 4.01-3.95 (m, 1H), 3.54 (s, 1H), 2.80-2.76 (m, 1H), $2.67(\mathrm{dd}, J=7.6,4.4 \mathrm{~Hz}, 1 \mathrm{H}), 2.30$ $(\mathrm{s}, 3 \mathrm{H}), 1.09(\mathrm{~d}, J=6.4 \mathrm{~Hz}, 3 \mathrm{H}) ;{ }^{13} \mathrm{C}$ NMR $\left(100 \mathrm{MHz} \mathrm{CDCl}_{3}\right)$ $\delta 148.3,135.9,134.0,133.5,132.2,129.5,129.4,129.1,128.1$, $124.6\left(\mathrm{q},{ }^{1} J_{C F}=283.0 \mathrm{~Hz}\right), 124.0,123.7,122.2,102.8,77.3(\mathrm{q}$, $\left.{ }^{2} J_{C F}=31.0 \mathrm{~Hz}\right), 60.0,55.8,54.6,21.0,20.7$; HRMS calcd for 
$[\mathrm{M}] \mathrm{C}_{23} \mathrm{H}_{21} \mathrm{~F}_{3} \mathrm{~N}_{2} \mathrm{O}_{4}$ 446.1453, found 446.1456.

Compound 14: light brown solid, mp $63-64{ }^{\circ} \mathrm{C}$; IR (neat) 3428, 2937, 1708, 1533, 1349, 1247, 1170, 1035, 836, $801 \mathrm{~cm}^{-1}$; ${ }^{1} \mathrm{H}$ NMR $\left(400 \mathrm{MHz}, \mathrm{CDCl}_{3}\right) \delta 8.19-8.14(\mathrm{~m}, 2 \mathrm{H}), 7.59-7.57$ $(\mathrm{m}, 1 \mathrm{H}), 7.51-7.47(\mathrm{~m}, 1 \mathrm{H}), 7.06-7.03(\mathrm{~m}, 2 \mathrm{H}), 6.77-6.75(\mathrm{~m}$, $2 \mathrm{H}), 6.33(\mathrm{~s}, 1 \mathrm{H}), 4.91-4.82(\mathrm{~m}, 1 \mathrm{H}), 4.23-4.20(\mathrm{~m}, 1 \mathrm{H}), 4.02-$ $3.95(\mathrm{~m}, 1 \mathrm{H}), 3.77(\mathrm{~s}, 3 \mathrm{H}), 3.59(\mathrm{~s}, 1 \mathrm{H}), 2.80-2.76(\mathrm{~m}, 1 \mathrm{H})$, 2.70-2.64 (m, 1H), 1.09 (d, J=6.4 Hz, 3H); ${ }^{13} \mathrm{C} \mathrm{NMR} \mathrm{(100} \mathrm{MHz,}$ $\left.\mathrm{CDCl}_{3}\right) \delta 158.0,148.3,135.9,133.9,133.5,129.5,129.3,128.9$, $127.7,124.7\left(\mathrm{q},{ }^{1} J_{C F}=282.0 \mathrm{~Hz}\right), 123.9,123.4,122.1,113.8$, 102.7, $77.2\left(\mathrm{q},{ }^{2} J_{C F}=31.0 \mathrm{~Hz}\right), 60.0,55.7,55.1,54.6,20.7$; HRMS calcd for $[\mathrm{M}] \mathrm{C}_{23} \mathrm{H}_{21} \mathrm{~F}_{3} \mathrm{~N}_{2} \mathrm{O}_{5} 462.1403$, found 462.1399 .

Acknowledgments. This research was supported by Basic Science Research Program through the National Research Foundation of Korea (NRF) funded by the Ministry of Education, Science and Technology (2009-0070538).

\section{References and Notes}

1. (a) Liedtke, A. J.; Keck, P. R. W. E. F.; Lehmann, F.; Koeberle, A.; Werz, O.; Laufer, S. A. J. Med. Chem. 2009, 52, 4968. (b) Michael,
J. P. Nat. Prod. Rep. 2005, 22, 603. (c) Liddell, J. R. Nat. Prod. Rep. 2002, 19, 773. (d) Atwell, G. J.; Fan, J.-Y.; Tan, K.; Denny, W. A. J. Med. Chem. 1998, 41, 4744. (e) Laufer, S. A.; Augustin, J.; Dannhardt, G.; Kiefer, W. J. Med. Chem. 1994, 37, 1894. (f) Gruters, R. A.; Neefjes, J. J.; Tersmette, M.; de Goede, R. A. Y.; Tulp, A.; Huisman, H. G.; Miedema, F.; Ploegh, H. L. Nature 1987, 330, 74. (g) Zalkow, L. H.; Glinski, J. A.; Gelbaum, L. T.; Fleischmann, T. J.; McGowan, L. S.; Gordon, M. M. J. Med. Chem. 1985, 28, 687.

2. Purser, S.; Moore, P. R.; Swallow, S.; Gouverneuer, V. Chem. Soc. Rev. 2008, 37, 320 .

3. Bae, J.-Y.; Lee, H.-J., Youn, S.-H.; Kwon, S.-H.; Cho, C.-W. Org. Lett. 2010, 12, 4352.

4. (a) Schneider, C.; Gueyrard, D.; Joseph, B.; Goekjian, P. G. Tetrahedron 2009, 65, 5427. (b) Guadagnin, R. C.; Suganuma, C. A.; Singh, F. V.; Vieira, A. S.; Cella, R.; Stefani, H. A. Tetrahedron Lett. 2008, 49, 4713. (c) Taylor, R. H; Felpin, F.-X. Org. Lett. 2007, 9, 2911.

5. For recent reviews of two sequential cross-coupling reactions of multiple halogenated heterocycles, see: (a) Schnürch, M.; Flasik, R.; Khan, A. F.; Spina, M.; Mihovilovic, M. D.; Stanetty, P. Eur. J. Org. Chem. 2006, 3283. (b) Schröter, S.; Stock, C.; Bach, T. Tetrahedron 2005, 61, 2245.

6. (a) Hung, N. T.; Hussain, M.; Malik, I.; Villinger, A.; Langer, P. Tetrahedron Lett. 2010, 51, 2420. (b) Dang, T. T.; Ahmad, R.; Dang, T. T.; Reinke, H.; Langer, P. Tetrahedron Lett. 2008, 49, 1698. (c) Schröter, S.; Bach, T. Synlett 2005, 1957. 